University of Nebraska - Lincoln

DigitalCommons@University of Nebraska - Lincoln

9-9-2019

\title{
Generation and stability of structurally imprinted target skyrmions in magnetic multilayers
}

\author{
Noah Kent \\ Lawrence Berkeley National Laboratory \\ Robert Streubel \\ Lawrence Berkeley National Laboratory, streubel@unl.edu \\ Charles Henri Lambert \\ ETH Zürich \\ Alejandro Ceballos \\ University of California, Berkeley \\ Soong Gun Je \\ Lawrence Berkeley National Laboratory
}

See next page for additional authors

Follow this and additional works at: https://digitalcommons.unl.edu/physicsstreubel

Part of the Atomic, Molecular and Optical Physics Commons, Condensed Matter Physics Commons, and the Other Physics Commons

Kent, Noah; Streubel, Robert; Lambert, Charles Henri; Ceballos, Alejandro; Je, Soong Gun; Dhuey, Scott; Im, Mi Young; Büttner, Felix; Hellman, Frances; Salahuddin, Sayeef; and Fischer, Peter, "Generation and stability of structurally imprinted target skyrmions in magnetic multilayers" (2019). Robert Streubel Papers. 7.

https://digitalcommons.unl.edu/physicsstreubel/7

This Article is brought to you for free and open access by the Research Papers in Physics and Astronomy at DigitalCommons@University of Nebraska - Lincoln. It has been accepted for inclusion in Robert Streubel Papers by an authorized administrator of DigitalCommons@University of Nebraska - Lincoln. 


\section{Authors}

Noah Kent, Robert Streubel, Charles Henri Lambert, Alejandro Ceballos, Soong Gun Je, Scott Dhuey, Mi Young Im, Felix Büttner, Frances Hellman, Sayeef Salahuddin, and Peter Fischer 


\section{Generation and stability of structurally imprinted target skyrmions in magnetic multilayers}

Cite as: Appl. Phys. Lett. 115, 112404 (2019); https://doi.org/10.1063/1.5099991

Submitted: 14 April 2019 • Accepted: 28 August 2019 • Published Online: 12 September 2019

Doah Kent, (iD Robert Streubel, Charles-Henri Lambert, et al.
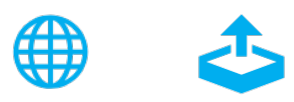

\section{ARTICLES YOU MAY BE INTERESTED IN}

Perspective: Magnetic skyrmions-Overview of recent progress in an active research field Journal of Applied Physics 124, 240901 (2018); https://doi.org/10.1063/1.5048972

The design and verification of MuMax3

AIP Advances 4, 107133 (2014); https://doi.org/10.1063/1.4899186

A single-lens universal interferometer: Towards a class of frugal optical devices

Applied Physics Letters 115, 111102 (2019); https://doi.org/10.1063/1.5108587

\section{Challenge us.}

What are your needs for periodic signal detection?

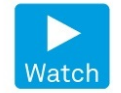

Zurich

Instruments

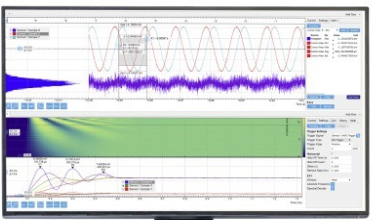

0 


\title{
Generation and stability of structurally imprinted target skyrmions in magnetic multilayers
}

Cite as: Appl. Phys. Lett. 115, 112404 (2019); doi: 10.1063/1.5099991

Submitted: 14 April 2019 - Accepted: 28 August 2019 .

Published Online: 12 September 2019

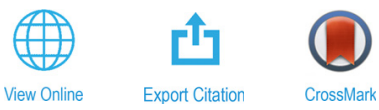

Noah Kent, ${ }^{1,2}$ iD Robert Streubel, ${ }^{1}$ iD Charles-Henri Lambert, ${ }^{3,4}$ Alejandro Ceballos, ${ }^{5}$ Soong-Gun Je, ${ }^{1,6}$ Scott Dhuey,

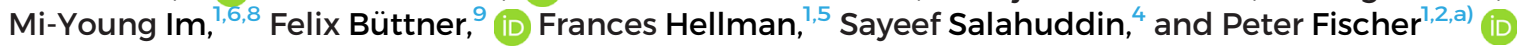

\author{
AFFILIATIONS \\ ${ }^{7}$ Materials Sciences Division, Lawrence Berkeley National Lab, 1 Cyclotron Rd., Berkeley, California 94720, USA \\ ${ }^{2}$ Physics Department, University of California Santa Cruz, 1156 High St., Santa Cruz, California 95064, USA \\ ${ }^{3}$ Department of Materials, ETH Zurich, Hönggerbergring 64, 8093 Zürich, Switzerland \\ ${ }^{4}$ Department of Electrical Engineering and Computer Sciences, UC Berkeley, 2626 Hearst Ave., Berkeley, California 94720, USA \\ ${ }^{5}$ Physics Department, University of California Berkeley, 366 LeConte Hall, Berkeley, California 94720, USA \\ ${ }^{6}$ Department of Emerging Materials Science, DGIST, 333, Techno Jungang-Daero, Hyeonpung-Myeon, Dalseong-Gun, \\ Daegu 711-873, South Korea \\ ${ }^{7}$ Molecular Foundry, Lawrence Berkeley National Lab, 1 Cyclotron Rd., Berkeley, California 94720, USA \\ ${ }^{8}$ Physics School of Material Science and Engineering, KIST-UNIST Ulan Center for Convergent Materials, \\ Ulsan National Institute of Science and Technology, 50, UNIST-gil, Ulsan 44919, South Korea \\ ${ }^{9}$ MIT Department of Materials Science and Engineering, 182 Memorial Dr., Cambridge, Massachusetts 02142, USA
}

${ }^{a)}$ Author to whom correspondence should be addressed: PJFischer@lbl.gov

\begin{abstract}
Target Skyrmions (TSks) are extended topological spin textures with a constant chirality where the rotation of the $\mathrm{z}$ component of the magnetization is larger than $\pi$. TSks have topological charge 1 or 0 , if the $z$ component of the magnetization $\mathrm{M}_{\mathrm{z}}$ goes through a rotation of $\mathrm{n} \pi$ where $\mathrm{n}$ is an odd or even integer, respectively. TSks with a rotation of the $\mathrm{z}$ component of up to $4 \pi$ have been imaged via high spatial resolution element-specific $\mathrm{x}$-ray imaging. The TSks were generated by weakly coupling $30 \mathrm{~nm}$ thin Permalloy $\left(\mathrm{Ni}_{80} \mathrm{Fe}_{20}, \mathrm{PY}\right)$ disks with a $1 \mu \mathrm{m}$ diameter to asymmetric ( $\operatorname{Ir} 1 \mathrm{~nm} /$ Co $1.5 \mathrm{~nm} / \mathrm{Pt} 1 \mathrm{~nm}$ ) $\times 7$ multilayers that exhibit Dzyaloshinskii-Moriya interaction. The PY disks stabilize the TSks in the multilayers due to reduced stray field energy and enforced circular symmetry from pinning of domain walls at the edges of the disks. Upon applying external magnetic fields, it is the skyrmion core at the center that ensures stability of the TSk, whereas the collapse of the extended structures in the TSk does not depend on the topological charge.
\end{abstract}

Published under license by AIP Publishing. https://doi.org/10.1063/1.5099991

Skyrmions, which are topologically protected magnetic spin textures and can result from inversion symmetry breaking Dzyaloshinskii Moriya interaction (DMI), are scientifically interesting and studied as potential building blocks in future spintronics devices, due to their stability, size, and mobility. ${ }^{1}$ They can be described by a topological charge, which is defined by $N_{s k}=\frac{1}{4 \pi} \iint d^{2} r \bar{m} \times\left(\frac{\partial \bar{m}}{\partial \mathrm{x}} \times \frac{\partial \bar{m}}{\partial y}\right)$, with $\bar{m}$ being the magnetization; for a classic skyrmion, $N_{s k}=1$. This topological charge provides stability because in order to destroy a skyrmion, and therefore change the topological charge, all collapse paths require a transition through an energetically highly unfavorable magnetic state, where the topology of the system is no longer defined. ${ }^{2,3}$ Ignoring boundary destruction, in most cases, this transition is mediated by a Bloch point. Even though skyrmions were predicted to exist in a large phase space, ${ }^{4}$ they were initially observed only at high magnetic fields and low temperatures. ${ }^{5}$ However, recently, several experiments have shown that skyrmions can also be found at room temperature and in zero magnetic field. ${ }^{6-11}$

Besides finding skyrmions in materials with intrinsic inversion symmetry breaking, shape anisotropy, interlayer exchange coupling, or stray field interaction between structurally confined magnetic heterostructures can serve as a mechanism to generate and stabilize topological spin structures. The latter has shown to enhance skyrmion stability, ${ }^{12}$ generate local topological spin textures with varying charge, ${ }^{13}$ and locally modify properties of magnetic films. ${ }^{14,15}$ 
Other magnetic skyrmionlike structures with an integer topological charge, such as stretched skyrmions, chiral bubbles, chiral horse shoe domains, and target skyrmions (TSks), have been theoretically predicted and experimentally confirmed. Target skyrmions (TSks), originally named $n-\pi$ vortices, have been predicted to be stable in extended films with DMI; ${ }^{16,36}$ TSks are extended skyrmionlike spin textures with more than a one $\pi$ rotation of the $\mathrm{z}$ component of magnetization $\mathrm{M}_{\mathrm{z}}$. The classic skyrmion spans one Bloch sphere, corresponding to a rotation of one- $\pi$. Recent experiments have found TSks $^{17,18,35}$ at low temperatures and/or in confined structures. However, thus far, there have been no observations of target skyrmion states with more than $2 \pi$ rotations of $\mathrm{M}_{\mathrm{z}}$.

The stability of classic skyrmions and the role of topology as a function of magnetic field and temperature have been explored both theoretically and experimentally; ${ }^{19-21}$ such a treatment for target skyrmions is lacking especially experimentally due to the dearth of observed TSks.

Here, we report the experimental observation of target skyrmions at room temperature, stabilized by DMI and stray field energies, with up to a $4 \pi$ rotation of the $\mathrm{z}$ component of the magnetization, and study their stability as a function of externally applied magnetic field and topological charge.

To synthesize TSks, we deposited antisymmetric $\mathrm{Ta}[3]+[\operatorname{Ir}[1] /$ $\mathrm{Co}[1.5] / \mathrm{Pt}[1]] \times 7+\mathrm{Pt}[2](\mathrm{nm})$ multilayers onto $100 \mathrm{~nm}$ thin polycrystalline silicon nitride $\left(\mathrm{Si}_{3} \mathrm{~N}_{4}\right)$ membranes to allow for high resolution magnetic imaging with transmission soft X-ray microscopy using $\mathrm{X}$-ray magnetic circular dichroism (XMCD) as element-specific contrast. These multilayers have shown to enable stable individual skyrmions at room temperature and in zero external field ${ }^{9,11}$ due to large interfacial DMI. The multilayers were deposited by DC magnetron sputtering at rates of $(0.01-0.05) \mathrm{nm} / \mathrm{s}$ (base pressure: $5 \times 10^{-8}$ Torr; argon pressure: $3.5 \mathrm{mTorr}$ ). Atomic force microscopy (AFM) showed a local film roughness of less than $0.1 \mathrm{~nm}$ (RMS), and magnetic hysteresis loops $[\mathrm{M}(\mathrm{H})]$ derived from vibrating sample magnetometry (VSM) matched well with analogous $\mathrm{Ir} / \mathrm{Co} / \mathrm{Pt}$ multilayers. ${ }^{11}$

On top of these multilayers, $30 \mathrm{~nm}$ thin Permalloy (PY, $\mathrm{Fe}_{80} \mathrm{Ni}_{20}$ ) films were grown via electron beam evaporation and patterned into disks with a diameter of $1 \mu \mathrm{m}$ using state-of-the-art electron beam lithography [Fig. 1(a)]. PY disks with these dimensions are known to exhibit a vortex state ${ }^{22}$ with an out-of-plane magnetization only at its core center. ${ }^{23}$ Further, this azimuthal symmetry in the spin texture appeared to be crucial for the imprinting as it favors the nucleation of skyrmions and target skyrmions.

Magnetic imaging was performed using the full-field transmission soft X-ray microscope (MTXM, BL 6.1.2) at the Advanced Light Source in Berkeley, CA. ${ }^{24}$ The XMCD contrast is sensitive to the magnetization component along the photon propagation direction and allows us to discriminate between the spin textures in the various elements, i.e., in the PY disk and the $\mathrm{Pt} / \mathrm{Co} / \mathrm{Ir}$ film by recording images at element-specific $\mathrm{x}$-ray absorption energies, e.g., $707 \mathrm{eV}$ for the $\mathrm{Fe}_{\mathrm{L}_{3}}$ and $778 \mathrm{eV}$ for the $\mathrm{Co} \mathrm{L}_{3}$ edges, respectively. Recording the spin textures in external magnetic fields allows us to study their stability.

The domain pattern in the multilayer [Fig. 1(b)] recorded at the Co $\mathrm{L}_{3}$ edge shows for the out-of-plane magnetic component the wellknown meanderlike pattern along with skyrmions that are characteristic to $\mathrm{Ir} / \mathrm{Co} / \mathrm{Pt}$ multilayers. ${ }^{11}$ a)
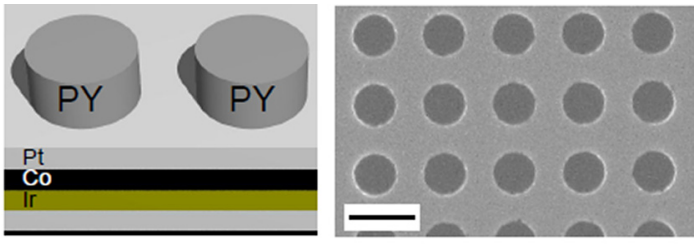

b)
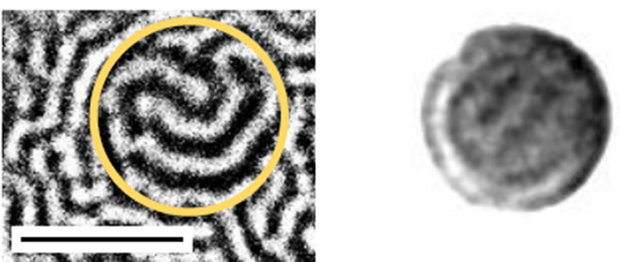

FIG. 1. (a) Left: Schematics of Permalloy disks on top of the asymmetric Pt/Co/lt multilayer. Right: SEM image of the sample. Scale bar $2 \mu \mathrm{m}$. (b) Left: MTXM image recorded at the $\mathrm{Co}_{3}$ edge showing the domain pattern in the multilayer in zero magnetic field. The yellow circle indicates the location of the PY disk. Right: MTXM image recorded at the $\mathrm{Fe}_{L_{3}}$ edge showing a weak out-of-plane contrast in the PY disk. Scale bar for both images is $1 \mu \mathrm{m}$.

Outside the PY disks, the domains in the multilayer reveal a periodicity of $(180 \pm 20) \mathrm{nm}$ which increases to $(240 \pm 20) \mathrm{nm}$ underneath the disks (Fig. 2). The periodicity increases since PY has a higher magnetic susceptibility than vacuum, which reduces the energy of the stray fields generated by the multilayer film. This can be validated by an analytical approach ${ }^{25}$ which finds energy minima for a Hamiltonian of magnetic multilayers with DMI. Using experimental values for anisotropy $\left(10^{7} \mathrm{~J} / \mathrm{m}^{3}\right)$, number of repeats of the multilayer (7), saturation magnetization $\mathrm{M}_{\mathrm{s}}\left(1.1 \times 10^{-3} \mathrm{~A} / \mathrm{nm}\right)$, and literature values for the interfacial DMI $\left(1.4 \times 10^{-21} \mathrm{~J} / \mathrm{nm}^{2}\right)^{9}$ and exchange constant $\left(10^{-21} \mathrm{~J} / \mathrm{nm}\right),{ }^{34}$ we calculated the width of the stripe domain periodicity in the magnetic multilayers by using these parameters and following the form and approach of Ref. 25. The derived result for the domain periodicity in the multilayers outside the disks gave a value $176.6 \mathrm{~nm}$, which is in excellent agreement with the experimental data, $180 \pm 20 \mathrm{~nm}$. To modify the strength of the surface stray field energy, a numerical prefactor was attached to the surface stray field energy term in the Hamiltonian of Ref. 27 and varied to reproduce our experimental observations. The observed increase in domain periodicity underneath the disks could be reproduced with an effective stray field energy reduction of $17 \%$. A varying anisotropy could also lead to a change in domain periodicity. However, since the change in anisotropy between two coupled layers depends on the magnetization direction of the imprinting layer ${ }^{27}$ the anisotropy modification, and hence domain periodicity, would not be constant underneath the disks. We observe a uniform shift in domain periodicity, and so a change in anisotropy cannot be responsible for the shift in domain periodicity.

Images taken at the $\mathrm{Fe}_{3}$ edge [Fig. 1(b), right] show a weak contrast, indicating a slight canting of the primarily in-plane moments at the locations of the PY disk. Although interlayer exchange coupling has shown to penetrate through $8 \mathrm{~nm}$ of $\mathrm{Pd},{ }^{14}$ a similar coupling through the top layer of $3 \mathrm{~nm}$ Pt seems rather unlikely. ${ }^{26}$ Micromagnetic simulations using $\mathrm{OOMMF}^{28}$ (cell size $1 \mathrm{~nm} \times 1 \mathrm{~nm} \times 1 \mathrm{~nm}$ ) were performed with a film that is weakly coupled (interlayer exchange coupling constant $=0.003 \mathrm{~mJ} / \mathrm{m}^{2}$ ) to a $1 \mu \mathrm{m}$ diameter, $30 \mathrm{~nm}$ thick PY disk. The magnetic properties of the film used in the simulation matched the experimental 


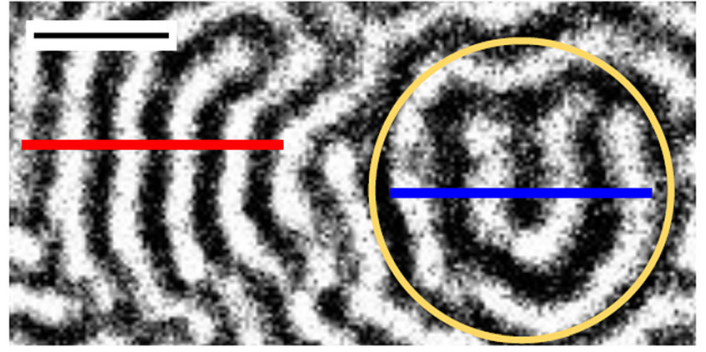

XMCD Signal Under Disks vs Free Film $\underset{\text { Under Disk }}{\text { Free Film }}$
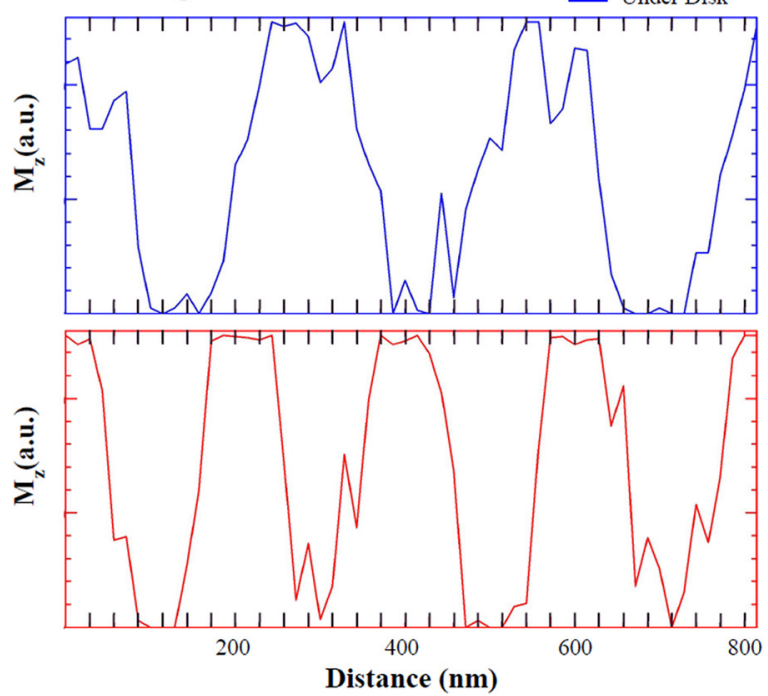

FIG. 2. MTXM intensity profiles showing an increase in domain width underneath the PY disks compared to the free film. Intensity profiles were taken along the red (free film) and blue (underneath disk) lines.

$\mathrm{Ir} / \mathrm{Co} / \mathrm{Pt}$ multilayers except for a reduced thickness ( $3 \mathrm{~nm}$ to reduce computation time). Standard OOMMF values for Permalloy (exchange constant $10^{-21} \mathrm{~J} / \mathrm{nm}, \mathrm{M}_{\mathrm{s}} 8 \times 10^{-7} \mathrm{~A} / \mathrm{nm}$, and no anisotropy) were used for the disk. Previous reports, as well our current simulations, have shown that the magnetization of the soft PY disks can be tilted out-of-plane by the stray fields that emanate from the film underneath. ${ }^{14}$ However, these simulations do not show a reciprocal canting of the film underneath into an in-plane configuration. The vortex spin configuration has no stray fields that would tilt the multilayers slightly in-plane; the only stray fields originate from the vortex core, which is laterally confined to $<10 \mathrm{~nm},{ }^{23}$ and furthermore point along the magnetization of the perpendicular magnetic anisotropy (PMA) multilayer film. A detailed study of those unusual spin configurations goes beyond the scope of this letter but is the subject of ongoing research and will be published elsewhere.

The coupling between a hard and a soft magnetic material can result in a reduction of anisotropy in the hard magnetic material without necessarily inducing a tilt in the magnetization..$^{20,27}$ By simulating a $1 \mu \mathrm{m}$ diameter, $30 \mathrm{~nm}$ thick PY disk in a forced out-of-plane magnetic configuration and looking at the time derivative of the magnetization, one can see that the impetus for the magnetization of the disk to tilt in-plane increases with the increasing distance from the center of the disk. Hence, the effective in-plane anisotropy of the disk is the highest near the edge and the out-of-plane anisotropy of the multilayer is the lowest near the edge of the disk. Consequently, domain walls are pinned at the edge of the disk since the domain wall energy is proportional to the square root of the effective anisotropy for an out-of-plane magnetized film. A similar effect is responsible for stabilizing artificial skyrmion crystals in extended films. ${ }^{13,29,30}$

Figure 3 shows TSks that are stabilized underneath $1 \mu \mathrm{m}$ diameter Permalloy disks in zero field and at room temperature exhibiting up to a $4 \pi$ rotation of the $\mathrm{z}$ component of magnetization $\left(\mathrm{M}_{\mathrm{z}}\right)$. Each additional ring in these target skyrmions corresponds to an additional $\pi$ rotation of $\mathrm{M}_{\mathrm{z}}$. The number of additional rings seems to be only limited by the size of the disk. The TSks are stabilized in the extended film through a combination of domain wall pinning, a reduction in stray field energy, and the DMI of the extended film. To study the role of DMI, symmetric multilayers with low DMI consisting of $\mathrm{Ta}[3]+$ $[\mathrm{Pt}[1] / \mathrm{Co}[1] / \mathrm{Pt}[1]] \times 7+\mathrm{Pt}[2](\mathrm{nm})$, with identical PY disks on top as for the asymmetric stack were investigated. The symmetric sample displayed domain wall pinning at the edge of disks, as well as an increase in domain periodicity underneath the PY disks. However, no TSks were found, suggesting that a certain threshold value of DMI is requisite to stabilize TSks. This is not surprising, as the TSk state is energetically highly favorable with regard to DMI, due to the fact that all adjacent spins are slightly canted relative to each other. As the stray

a)

$$
\begin{gathered}
\mathrm{N}_{\mathrm{sk}}=1 \\
\Delta \mathrm{M}_{\mathrm{z}}=\pi
\end{gathered}
$$

$$
\mathrm{N}_{\mathrm{sk}}=0
$$$$
\Delta \mathrm{M}_{\mathrm{z}}=2 \pi
$$
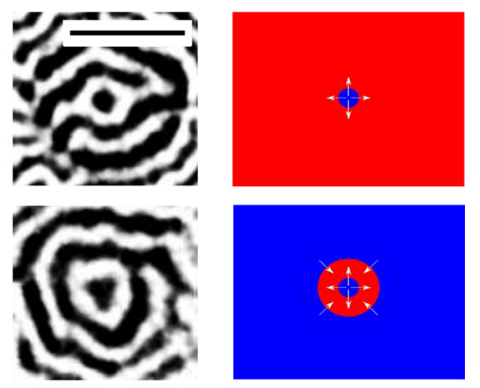

$$
\mathrm{N}_{\mathrm{sk}}=1
$$$$
\Delta \mathrm{M}_{\mathrm{z}}=3 \pi
$$
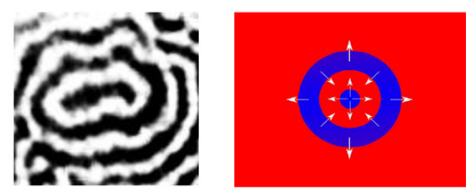

$$
\begin{gathered}
\mathrm{N}_{\mathrm{sk}}=0 \\
\Delta \mathrm{M}_{\mathrm{z}}=4 \pi
\end{gathered}
$$
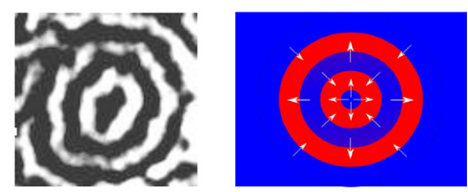

b)

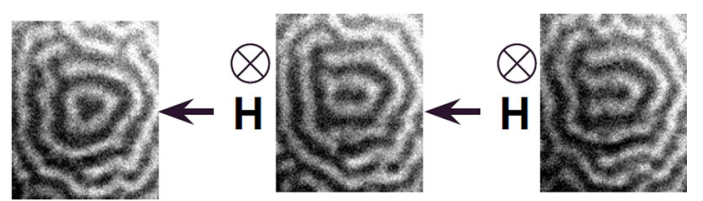

FIG. 3. (a) XMCD images of TSks at zero field paired with schematics highlighting the structure of the TSk. Red (blue) corresponds to the black (white) out-of-plane magnetization in the XMCD images; white arrows show the direction of the in-plane components. The scale bar is $1 \mu \mathrm{m}$. (b) Asymmetric domain expansion from out-ofplane magnetic field pulses with a small in-plane component, demonstrating constant Néel chirality. All images were taken at 0 field. 
field energy in TSks scales with the number of additional rings due to the increased area of each ring, we infer that TSks should be stabilized in an antisymmetric film underneath the PY disks with lower stray field energies.

Given the magnetization profile of a TSk, the topological charge is $^{31} N_{s k}=\frac{1}{2}[\cos \Theta(r)]_{0}^{\infty}[\Phi(\phi)]_{0}^{2 \pi}$, where $\Theta(\mathrm{r})$ is the angle between the magnetization and the $\mathrm{z}$ axis and $[\Phi(\phi)]_{0}^{2 \pi}$ is the vorticity, which can be defined as the number of rotations of the in-plane component of the magnetization goes through following a closed loop around the center at constant radius. For TSks, the topological charge depends on the number of rotations of the $\mathrm{z}$ component of the magnetization. It follows that in TSks, the topological charge toggles between 0 and 1 for each additional ring. It is worth noting that the spins at $r=\infty$ are defined as the magnetization which the skyrmions are surrounded by; a skyrmion has the spins at infinity antiparallel to its core, but a $2 \pi$ TSk, typically called a skyrmionium, has the spins at infinite parallel to its core (Fig. 3).

The topological charge of skyrmions and target skyrmions requires that the spin texture has a constant chirality. We confirmed a constant chirality in the multilayers imparted by their Néel type interfacial DMI through triggering an asymmetric bubble expansion in a pulsed magnetic field tilted in the out-of-plane direction. Figure 3(b) shows a uniform expansion of the domains in the direction of the inplane field asymmetry due to their Néel type DMI, proving that a constant chirality is maintained in the Co multilayers. ${ }^{3}$

To address the impact of the topological charge on the structural stability of a TSk against an external magnetic field, we examined a $2 \pi$ TSk, with $N_{s k}=0$, and a $3 \pi$ TSk with $N_{s k}=1$, as an external magnetic field was applied (Fig. 4). The external field was applied in the direction of the spins at infinity for each structure, i.e., "parallel" to the core of the $2 \pi$ TSk and "antiparallel" to the core of the $3 \pi$ TSk. When applying an external magnetic field to TSKs, the magnetization

a)

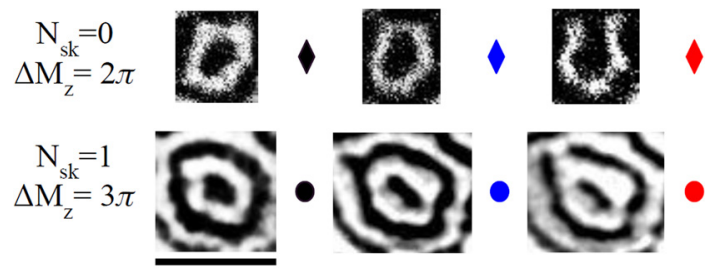

c)

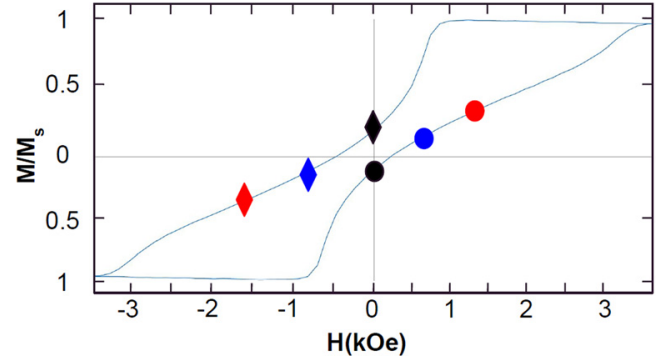

FIG. 4. (a) Evolution of a $2 \pi$ TSk in an external field aligned with the spins at infinite (in the direction of the black magnetization). (b) Same as in (a) but for a $3 \pi$ TSk with an external field aligned with the spins into the white direction. Scale bar: $1 \mu \mathrm{m}$. (c) $\mathrm{M}(\mathrm{H})$ of the multilayers measured with VSM. The black, blue, and red data points correspond to the images for the $2 \pi, 3 \pi$ TSk. The red markers are where the extended TSk structure collapses. pointing along the direction of the applied field expands, as shown by the blue markers in Fig. 4. Interestingly, both TSks collapse at almost the same field value of $1.6 \mathrm{kOe}$ (red marker Fig. 4), i.e., independent from their topological charge. The collapse of the $2 \pi$ TSk occurs similar to the collapse of a $2 \pi$ domain wall, which is in agreement with previous experimental observations in $2 \pi \mathrm{TSk},{ }^{17}$ leaving behind a chiral horseshoe domain with topological charge 1 . The outer ring in the $3 \pi$ TSk collapses in a similar manner to the $2 \pi$ TSk leaving a single skyrmion, with topological charge 1 , in the center surrounded by a chiral horseshoe domain. The structural collapse occurs at the same field for both TSks and is therefore independent from the initial topological charge. When the extended target skyrmion structure collapses, the topological charge changes by \pm 1 ; the charge 0 structure dissipates into a chiral horseshoe domain with its core magnetization opposite to that of the initial TSk $\left(N_{s k}=-1\right)$, and the charge 1 structure collapses to a single classic skyrmion surrounded by a chiral horseshoe domain $\left(N_{s k}=2\right)$. We can conclude that $2 \pi$ TSk-like structures, with no topological charge, will go through a topological change at a much lower external field when compared to topological charge 1 structures, as seen by the unperturbed central skyrmion in the $3 \pi \mathrm{TSk}$. We should note that at higher fields, it is very likely that these chiral horse shoe domains will form compact skyrmions, but due to the limitation of the magnetic field strength in this experiment, this could not yet be verified. Therefore, a $3 \pi$ TSk can be seen to be composed of a classic skyrmion, wrapped by a topologically trivial $2 \pi$ TSk. Topological stability in a real physical system relies on having an energy barrier so as to create topological change. The formation of a Bloch point which is necessary to unwind a skyrmion's core provides an additional energy barrier and topological protection, ${ }^{2,3}$ as opposed to topologically trivial structures, such as a $2 \pi$ TSk, which can unwind itself at much lower fields.

By weakly coupling PY disks to a multilayer film with DMI, we created TSks without modifying the chirality inherent in DMI films. The PY disks reduce the stray field energy and enforce azimuthal symmetry, stabilizing extended target skyrmions with varying topological charge in asymmetric multilayers. We observed that the overall structural stability of TSk is unrelated to their topological charge. This is due to the fact that stability induced by topological charge only applies to structures with nontrivial topological charge; target skyrmions are either topologically trivial or have a single skyrmion surrounded by a topologically trivial structure. This collapse is in agreement with simulations of pure DMI stabilized target skyrmions, ${ }^{35}$ even though, in general, the dynamic properties of skyrmions depend on the energies that stabilize them.

We anticipate that higher order $(5 \pi, 6 \pi$, etc.) TSks can be stabilized with larger diameter PY disks. Similarly, it remains to be seen whether other characteristics of skyrmions, such as the topological Hall effect and skyrmion Hall effect, depend on the topological charge in TSks. It is possible to envision spintronic devices that rely on the topological Hall effect and a controllable topological charge in TSk, which is a function the initial TSk state, and external magnetic field. Finally, TSks have been recently suggested as potential precursors for three dimensional topologically protected magnetic structures, such as hopfions. ${ }^{33,37}$

This work was funded by the U.S. Department of Energy, Office of Science, Office of Basic Energy Sciences, Materials Sciences and Engineering Division under Contract No. DE-AC0205-CH11231 within the NEMM program (MSMAG). 
The work at the Molecular Foundry was supported by the Office of Science, Office of Basic Energy Sciences, of the U.S. Department of Energy under Contract No. DE-AC02-05CH11231.

This research used resources of the Advanced Light Source, which is a DOE Office of Science User Facility under Contract No. DE-AC02-05CH11231.

The material synthesis was supported by the E3S center supported by the National Science Foundation.

M.-Y. Im acknowledges the support from the National Research Foundation (NRF) of Korea funded by the Ministry of Education, Science and ICT (Nos. 2018K1A4A3A03075584 and 2016M3D1A1027831) and by the DGIST R\&D program of the Ministry of Science, ICT and future Planning (18-BT-02).

\section{REFERENCES}

${ }^{1}$ A. Fert, N. Reyren, and V. Cros, Nat. Rev. Mater. 2, 17031 (2017).

${ }^{2}$ D. Cortés-Ortuño, W. Wang, M. Beg, R. A. Pepper, M. A. Bisotti, R. Carey, M.

Vousden, T. Kluyver, O. Hovorka, and H. Fangohr, Sci. Rep. 7(1), 4060 (2017).

${ }^{3}$ S. Rohart, J. Miltat, and A. Thiaville, Phys. Rev. B 93, 214412 (2016).

${ }^{4}$ U. K. Rößler, A. N. Bogdanov, and C. Pfleiderer, Nature 442, 797-801 (2006).

${ }^{5}$ S. Mühlbauer, B. Binz, F. Jonietz, C. Pfleiderer, A. Rosch, A. Neubauer, R. Georgii, and P. Böni, Science 323, 5916 (2009).

${ }^{6}$ G. Yu, A. Jenkins, X. Ma, S. A. Razavi, C. He, G. Yin, Q. Shao, Q. L. He, H. Wu, W. Li, W. Jiang, X. Han, X. Li, A. C. B. Jayich, P. K. Amiri, and K. L. Wang, Nano Lett. 18(2), 980-986 (2018).

${ }^{7}$ W. Jiang, P. Upadhyaya, W. Zhang, G. Yu, M. B. Jungfleisch, F. Y. Fradin, J. E. Pearson, Y. Tserkovnyak, K. L. Wang, O. Heinonen, S. G. E. te Velthuis, and A. Hoffmann, Science 349(6245), 283 (2015).

${ }^{8}$ S. Woo, K. Litzius, B. Krüger, M.-Y. Im, L. Caretta, K. Richter, M. Mann, A. Krone, R. M. Reeve, M. Weigand, P. Agrawal, I. Lemesh, M.-A. Mawass, P. Fischer, M. Kläui, and G. S. D. Beach, Nat. Mater. 15, 501 (2016).

${ }^{9}$ C. Moreau-Luchaire, C. Moutafis, N. Reyren, J. Sampaio, C. A. F. Vaz, N. Van Horne, K. Bouzehouane, K. Garcia, C. Deranlot, P. Warnicke, P. Wohlhüter, J. M. George, M. Weigand, J. Raabe, V. Cros, and A. Fert, Nat. Nanotechnol. 11, 444 (2016).

${ }^{10}$ O. Boulle, J. Vogel, H. Yang, S. Pizzini, D. de Souza Chaves, A. Locatelli, T. O. Menteş, A. Sala, L. D. Buda-Prejbeanu, O. Klein, M. Belmeguenai, Y. Roussigné, A. Stashkevich, S. M. Chérif, L. Aballe, M. Foerster, M. Chshiev, S. Auffret, I. M. Miron, and G. Gaudin, Nat. Nanotechnol. 11, 449-454 (2016).

${ }^{1}$ A. Soumyanarayanan, M. Raju, A. L. G. Oyarce, A. K. C. Tan, M.-Y. Im, A. P. Petrović, P. Ho, K. H. Khoo, M. Tran, C. K. Gan, F. Ernult, and C. Panagopoulos, Nat. Mater. 16, 898 (2017).

${ }^{12}$ H. Z. Wu, B. F. Miao, L. Sun, D. Wu, and H. F. Ding, Phys. Rev. B 95, 174416 (2017).
${ }^{13}$ J. Li, A. Tan, K. W. Moon, A. Doran, M. A. Marcus, A. T. Young, E. Arenholz, S. Ma, R. F. Yang, C. Hwang, and Z. Q. Qiu, Nat. Commun. 5, 4704 (2014).

${ }^{14}$ R. Streubel, L. Han, M.-Y. Im, F. Kronast, U. K. Rößler, F. Radu, R. Abrudan, G. Lin, O. G. Schmidt, P. Fischer, and D. Makarov, Sci. Rep. 5, 8787 (2015).

${ }^{15}$ R. Streubel, P. Fischer, M. Kopte, O. G. Schmidt, and D. Makarov, Appl. Phys. Lett. 107, 112406 (2015).

${ }^{16}$ A. Bogdanov and A. Hubert, J. Magn. Magn. Mater. 195, 182-192 (1999).

${ }^{17}$ S. Zhang, F. Kronast, G. van der Laan, and T. Hesjedal, Nano Lett. 18, 1057-1063 (2018).

${ }^{18}$ F. Zheng, H. Li, S. Wang, D. Song, C. Jin, W. Wei, A. Kovács, J. Zang, M. Tian, Y. Zhang, H. Du, and R. E. Dunin-Borkowski, Phys. Rev. Lett. 119, 197205 (2017).

${ }^{19}$ F. Büttner, I. Lemesh, and G. S. D. Beach, Sci. Rep. 8, 4464 (2018).

${ }^{20}$ R. Tomasello, K. Y. Guslienko, M. Ricci, A. Giordano, J. Barker, M. Carpentieri, O. Chubykalo-Fesenko, and G. Finocchio, Phys. Rev. B 97, 060402(R) (2018).

${ }^{21} \mathrm{~N}$. Romming, A. Kubetzka, C. Hanneken, K. von Bergmann, and R. Wiesendanger, Phys. Rev. Lett. 114, 177203 (2015).

${ }^{22}$ T. Shinjo, T. Okuno, R. Hassdorf, K. Shigeto, and T. Ono, Science 289, 930-932 (2000).

${ }^{23}$ P. Fischer, M.-Y. Im, S. Kasai, K. Yamada, T. Ono, and A. Thiaville, Phys. Rev. B 83, 212402 (2011).

${ }^{24}$ P. Fischer, D.-H. Kim, W. Chao, J. A. Liddle, E. H. Anderson, and D. T. Attwood, Mater. Today 9, 26 (2006).

${ }^{25}$ I. Lemesh, F. Büttner, and G. S. D. Beach, Phys. Rev. B 95, 174423 (2017).

${ }^{26}$ J. Moritz, F. Garcia, J. C. Toussaint, B. Dieny, and J. P. Nozières, Europhys. Lett. 65, 123-129 (2004).

${ }^{27}$ L. Fallarino, V. Sluka, B. Kardasz, M. Pinarbasi, A. Berger, and A. D. Kent, Appl. Phys. Lett. 109, 082401 (2016).

${ }^{28}$ M. J. Donahue and D. G. Porter, OOMMF User's Guide, version 1.0, Interagency Report NISTIR 6376, National Institute of Standards and Technology, Gaithersburg, MD (1999), available at http://math.nist.gov/oommf.

${ }^{29}$ D. A. Gilbert, B. B. Maranville, A. L. Balk, B. J. Kirby, P. Fischer, D. T. Pierce, J. Unguris, J. A. Borchers, and K. Liu, Nat. Commun. 6, 8462 (2015).

${ }^{30}$ L. Sun, R. X. Cao, B. F. Miao, Z. Feng, B. You, D. Wu, W. Zhang, A. Hu, and H. F. Ding, Phys. Rev. Lett. 110, 167201 (2013).

${ }^{31}$ H.-B. Braun, Adv. Phys. 61, 1-116 (2012).

${ }^{32}$ S.-G. Je, D.-H. Kim, S.-C. Yoo, B.-C. Min, K.-J. Lee, and S.-B. Choe, Phys. Rev. B 88, 214401 (2013).

${ }^{33}$ P. Sutcliffe, J. Phys. A 51, 375401 (2018).

${ }^{34}$ C. Kittel, Introduction to Solid State Physics, 7 th ed. (John Wiley and Sons, Inc., 1996).

${ }^{35}$ D. Cortés-Ortuño, N. Romming, M. Beg, K. von Bergmann, A. Kubetzka, O. Hovorka, H. Fangohr, and R. Wiesendanger, Phys. Rev. B 99, 214408 (2019).

${ }^{36}$ M. Beg, R. Carey, W. Wang, D. Cortés-Ortuño, M. Vousden, M. A. Bisotti, M. Albert, D. Chernyshenko, O. Hovorka, R. Stamps, and H. Fangohr, Sci. Rep. 5, 17137 (2015).

${ }^{37}$ Y. Liu, R. Lake, and J. Zang, Phys. Rev. B 98, 174437 (2018). 